\title{
New Bounds On Rake Structures for DS-CDMA Over Frequency-Selective Rayleigh Fading Channels
}

\author{
Sergio Calvo, Gregori Vazquez \\ Polytechnic University of Catalonia. Dpt. of Signal Theory and Communications \\ c/ Gran Capità s/n, 08034 Barcelona, SPAIN. Tel: 34-3-4016440 Fax: 34-3-4016447 \\ e-mail: gregori@gps.tsc.upc.es, sergio@gps.tsc.upc.es
}

\begin{abstract}
An upper bound is derived for the probability of error in an asynchronous binary direct-sequence spread-spectrum multiple-access communications system operating over frequency selective Rayleigh fading channels. A coherent RAKE receiver with predetection selective diversity combining is considered. The performance of a multipathcombinig receiver is determined for the case of multiple interfering transmitters. Furthermore, the performance of the system is determined in terms of parameters of the signature sequences. These parameters can be used as guides in selecting sequences for the system. The bounds agree with the exponential portion of a normal distribution in which the interfering interference components subtract from the signal amplitude. The results obtained are verified by simulation.
\end{abstract}

\section{Introduction}

In this paper, we consider the performance analysis of the multipath-combinig receiver also called RAKE receiver. The analysis applies to systems that use binary phaseshift-keyed (BPSK) modulation. We consider a multipathcombinig receiver and determine the performance of the system for the case of multiple interfering transmitters that use different PN sequences but having a small factor of correlation.

The system designer use to assume that the limiting corrupting signal has a Gaussian distribution. This assumption can no longer be justified, and the interference in multiple-access schemes will not show a Gaussian characteristics. Although a small thermal noise power will be present, it is necessary to consider the joint effect of the Gaussian-distributed thermal noise and the nonGaussian distributed interference.

The analysis will be based on the well known Chernoff bound. This analysis requires only the evaluation

This work has been partially supported by the Spanish Research Council (CYCIT) under grant TIC95-1022-C05-01 or bounding of the moment-generating function of the additive interference. The bound is expressed in terms of a parameter that is the unique solution of an equation containing the derivative of the moment generating function of the interference. The bound is tight for high SNRs, which is the region of interest in most mobile communications systems.

\section{A System and Channel Model}

The system of communications proposed for this study is shown in figure (1).

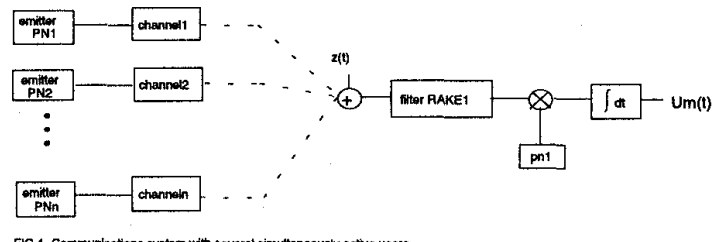

The k-th transmitted signal for a binary DS CDMA system with BPSK modulation and arbitrary chip waveform can be expressed as

$s_{k}(t)=\sqrt{2} P_{k} \sum_{i} b_{k}(i) \psi(t) u_{k}(t-i T)$

where $P_{k}$ is the power in each $K$-th transmitted signal, $u_{k}(t)$ is the signature waveform and $b_{k}(i)$ the $i-t h$ symbol of the $\mathrm{k}$-th user. An equal power assumption, $\mathrm{P}_{\mathrm{k}}$, is made for convenience in the analysis. We assume that there are $\mathrm{N}$ code chips in each data symbol $\left(\mathrm{T}=\mathrm{NT}_{\mathrm{c}}\right)$ and the period of the signature sequence $\left(u_{k}(t)\right)$ is $N$. The received signal from a typical transmitter consists of a random number of paths of the transmitted signal. The delay, amplitude, and phase associated with each path are also random.

$r_{k}(t)=\sum_{n=1}^{L} \alpha_{n}(t) \exp \left(j \Phi_{n}(t)\right) s_{k}\left[t-\tau_{n}(t)\right]+z(t)$

In (2) the random variable $\mathrm{L}$ represents the number of paths of the $k$-th user. The random variables $\alpha_{n}(t), \tau_{n}(t)$, $\Phi_{n}(t)$, represent the amplitude, delay, and phase associated with the nth path of a signal from transmitter $k$. We 
consider the delay $\tau_{n}(t)$ as an integer of Tc. The term $z_{k}(t)$ represents the additive white Gaussian noise (AWGN) with complex spectral density No Watt/Hz.

We consider the fact that in a real communication system the links from each of the $\mathrm{K}$ active transmitters to the listening receiver are mutually independents. The random variables variables $\alpha_{n}(t), \tau_{n}(t), \Phi_{n}(t)$, and the data symbols, are assumed to form a set of mutually independent random variables. Also, each phase $\Phi_{n}(t)$ is assumed to be uniformly distributed on the interval $[0,2 \pi)$. We model the arrival of signal paths at receiver, $\tau_{n}(t)$, by a nonhomogeneous Poisson process with the arrival rate $P_{d}(x)$. The amplitudes of the signal paths, $\alpha_{n}(t)$, exhibit a Rayleigh distribution. The structure of the receiver is shown in figure (2).

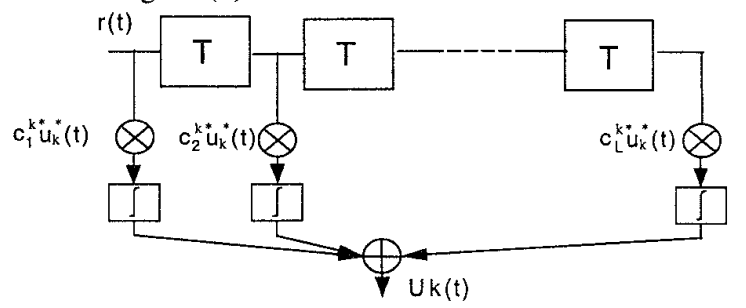

fig 2. Rake Structure

It employs a single tap delay line trough which is passed the received signal $r_{k}(t)$. The signal at each tap is correlated with $\left(c_{n}{ }^{k}\right)^{*}(t) u_{k}^{*}(t), n=1,2, \ldots, L$, where $u_{k}(t)$ is the PN sequence of the k-th desired user, $c_{n}^{k}=\alpha_{n}^{k} \exp \left(j \Phi_{n}^{k}\right)$ the impulse response of the channel associated to the desired user and (*) denotes complex conjugate.

\section{Evaluation of Error Probability Bound}

We shall now evaluate the performance of the RAKE receiver with simultaneous active users under the condition that the fading is sufficiently slow to allow us to estimate $c_{n}{ }^{k}(t)$ perfectly (without noise). Thus the decision variables may be expressed in the form

$$
\mathrm{U}_{\mathrm{k}}(\mathrm{i})=\Re \mathrm{e} \sum_{\mathrm{q}=1}^{\mathrm{L}}\left(\mathrm{c}_{\mathrm{q}}^{\mathrm{k}}\right)^{*} \int_{\mathrm{T}} \mathrm{r}(\mathrm{t}) \mathrm{u}_{\mathrm{k}}^{*}(\mathrm{t}-\mathrm{qT} \mathrm{T}) \mathrm{dt}
$$

Stationarity of the amplitudes paths of arrivals is assumed. The received signal can be expressed as follows

$$
\begin{aligned}
& r(t)=\sum_{i} b_{k}(i) \sum_{n=1}^{L} c_{n}^{k} u_{k}(t-n T c-i T)+ \\
& \sum_{n f=1}^{N f} \sum_{n f} b_{n f}(i) \sum_{p=1}^{L} c_{p}^{n f} u_{n f}\left(t-p T_{c}-i T\right)+z(t)
\end{aligned}
$$

where $u_{n f}(t)$ are the PN sequences and $b_{n f}(i)$ the symbols of the Nf users that share the same transmission band. Eq (3) can be expressed as:

$$
\begin{aligned}
& U_{k}=\Re e \sum_{q=1}^{L}\left|c_{q}^{k}\right|^{2} b \int_{k} \int_{\mathrm{T}}{ }_{k}(t-q T c) u_{k}^{*}(t-q T c) d t+ \\
& \Re e \sum_{n f=1}^{N f} \sum_{q=1 n=1}^{L} \sum_{q}^{L} \alpha_{n}^{k} \alpha_{n}^{n f} \operatorname{expj}\left(\Phi_{n}^{n f}-\Phi_{q}^{k}\right) b_{n f} \int_{T} u_{n f}(t-n T c) u_{k}^{*}(t-q T c) d t \\
& \mathrm{nf}=\mathrm{k}, \mathrm{q} \neq \mathrm{n} \\
& +\Re e \sum_{\mathrm{q}=1}^{\mathrm{L}} \alpha_{\mathrm{q}}^{\mathrm{k}} \exp \left(-\mathrm{j} \Phi_{\mathrm{q}}^{\mathrm{k}}\right) \int_{\mathrm{T}}^{\mathrm{z}}(\mathrm{t}) \mathrm{u}_{\mathrm{k}}^{*}(\mathrm{t}-\mathrm{q} \mathrm{Tc}) \mathrm{dt}
\end{aligned}
$$

When the signals are antipodal, a single decision variable suffices. Then, if consider the maximum cross-correlation value between the set of signature waveforms, Eq (5) can be simplifies to the following:

$$
\begin{aligned}
& U_{k}=R o b_{k} E \sum_{q=1}^{L}\left(\alpha_{q}^{k}\right)^{2}+\gamma E \sum_{n f=1}^{N f} \sum_{n=k, q \neq n}^{L} \sum_{q=1}^{L} \alpha_{q}^{k} \alpha_{n}^{n f} e^{j \Psi^{N f}, n}+\sum_{q=1}^{L} \alpha_{q}^{k} N_{q}^{k}(6) \\
& \text { with: }{ }_{N_{q}^{k}}=e^{-j \Phi^{k}} \int_{\mathrm{T}}^{\mathrm{q}(\mathrm{t}) \mathrm{u}_{\mathrm{k}}}{ }_{\mathrm{k}}^{(\mathrm{t}-\mathrm{qTC})} \text { and } \mathrm{e}^{\mathrm{j} \Psi_{\mathrm{q}, \mathrm{n}}^{\mathrm{nf}}}=\mathrm{e}^{\mathrm{j}\left(\Phi_{\mathrm{n}}^{\mathrm{nf}}-\Phi_{\mathrm{q}}^{\mathrm{k}}\right)} \mathrm{b}_{\mathrm{nf}} \\
& \gamma=\frac{2^{(m+1) / 2}+1}{2^{m}-1} \geq\left.\frac{\int_{u_{k}(t-m T c) u}(t-p T c) d t}{\int_{T} u_{k}(t) u_{k}(t) d t}\right|_{k=q, p \neq m}
\end{aligned}
$$

where $E=\int_{T} u_{k}(t) u_{k}^{*}(t) d t$ is the energy of the k-th signature waveform and the normalized cross-correlation bound holds for m-length Gold sequences. The interference will be modeled as follows:

$$
\eta=\gamma \mathrm{E} \sum_{\mathrm{i}=1}^{\mathrm{NfL} \xi_{\mathrm{i}} \cos \Theta_{i}}
$$

being $\xi_{\mathrm{i}}$ a random variable that results from the product of two independent Rayleigh distributed random variables, $\Theta_{i}$ is assumed to be uniformly distributed in the interval $[0,2 \pi)$ and $L^{\prime}=L(L-1)$. We define the total interference $z$ as $z=\eta+n$, that is, the multi-user interference plus de thermal noise term. As far as $z$ is the sum of two independent, zero-mean random variables, the Chernoff bound applies:

$\operatorname{Pr}(z>x) \leq \exp ^{-\lambda x} E[\exp \lambda n] E[\exp \lambda \eta]$ for all $\lambda>0$

after some manipulations (8) becomes:

$$
\operatorname{Pr}(z>x) \leq \exp ^{-\lambda x} \exp ^{-\frac{1}{2} \lambda^{2} \sigma_{n}^{2}} \prod_{i=1}^{\mathrm{NfL}} \operatorname{Io}\left(\lambda \xi_{i}\right) \text { for all } \lambda>0
$$

Equation (10) can be simplified by using exponential upper bounds to the order zero modified Bessel function

$\operatorname{Pr}(z>x) \leq \exp ^{-\lambda x} \exp ^{\frac{1}{2} \lambda^{2} \sigma^{2}} \exp ^{\sum_{i=1}^{N f L} \lambda \xi_{i}^{\prime}}$ for all $\lambda>0$

The optimal value of $\lambda$ which minimizes the right side of (10) is obtained by setting the derivative of the expression with respect to $\lambda$ to zero:

$$
\operatorname{Pr}(z>x) \leq \exp -\frac{\left(E \sum_{i=1}^{L} \alpha_{i}^{2}-E \gamma \sum_{i=1}^{L} \sum_{i}^{\prime N f}\right)^{2}}{\sigma_{n}^{2}}
$$




$$
\text { where } \begin{aligned}
\sigma_{n}^{2} & =E \text { No } \sum_{i=1}^{L} \alpha_{i}^{2} \text {. We define } \gamma_{b} \text { as: } \\
\gamma_{b} & =\frac{E \sum_{i=1}^{L} \alpha_{i}^{2}-E \gamma \sum_{i=1}^{L} \xi_{i}}{\sqrt{\sigma_{n}^{2}}}
\end{aligned}
$$

The final step in this derivation is to average the conditional error probability given in (11) over the fading channel statistics. Thus we evaluate the integral:

$$
\operatorname{Pe}=\int_{0}^{\infty} \operatorname{Pr}\left(\gamma_{b}\right) f\left(\gamma_{b}\right) d \gamma_{b}
$$

It is difficult to find the distribution function of (12), and with the resultant expression is not possible to obtain a closed expression of (13). Thus we consider the average of $\sigma_{n}^{2}$ instead the exact expression. Defining $E\left[\alpha_{n}^{2}\right]=2 \sigma^{2}$, the expression (12) reduces to:

$$
\gamma_{b}=\sqrt{\frac{E}{2 N_{0} \sigma^{2} L^{\prime}}}\left(\sum_{i=1}^{L_{i}^{\prime}} \alpha_{i}^{2}-\gamma \sum_{i=1}^{N f L} \xi_{i}\right)
$$

It can be shown that:

$$
f\left(\gamma_{b}\right)=\frac{e^{-\frac{\gamma_{b}}{2 \sigma^{2} \Omega}}}{\Omega(L-1) !(2 M-1) !\left(\sigma^{2}\right)^{L 2 M} L} \sum_{j}^{L-1}\left(j-\frac{L}{L}-1\right)\left(\frac{\gamma_{b}}{\Omega}\right)^{L-1-j} I(j+2 M)-\frac{1}{\beta^{j+2 M}}
$$

with $M=L$ 'Nf and $\beta=\frac{\gamma+2}{2 \gamma \sigma^{2}}$ when the variance of the amplitudes of the paths of arrival belonging to different users are equal. We can define $\Omega$ as:

$$
\Omega=\sqrt{\frac{E}{2 N o L^{\prime} \sigma^{2}}}
$$

Then, the error probability (13) results in

$$
P e \leq \mathrm{A} \sum_{j=0}^{L-1} \mathrm{Bj} \exp \left(\frac{1}{4 \sigma^{2} \Omega}\right)^{2} \frac{\sqrt{\pi}}{2}(L-1-j) ! \sum_{k=0}^{\infty} \frac{(-1)^{k}\left(\frac{1}{4 \sigma^{2} \Omega}\right)^{k}}{{ }_{2}{ }^{L-1-j-k} k !\left(1+\frac{L-1-j-k}{2}\right)}
$$

where the terms in this series correponding to $k=L^{\prime}-j+1, L^{\prime}-j+3$, $L^{\prime}-j+5, \ldots$ are understood to be zero.

$$
\begin{aligned}
& \mathrm{A}=\frac{1}{\Omega\left(L^{\prime}-1\right) !\left(2 L^{\prime} N f\right) !\left(\sigma^{2}\right)^{2 L^{\prime} M}{ }_{2}^{L^{\prime}} \gamma^{2 L^{\prime} N f}} \\
& B j=\left(\begin{array}{l}
L^{\prime}-1 \\
j
\end{array}\right) \Gamma\left(j+2 L^{\prime} N f\right) \frac{1}{\beta^{j+2 L^{\prime} N f}} \frac{1}{\Omega^{L^{\prime}-1-j}}
\end{aligned}
$$

\section{Numerical Results}

The format of the signal has the following parameters: bandwidth $\mathrm{W}=(100 \mathrm{nsec})^{-1}=10 \mathrm{Mhz}$; spread factor $\mathrm{TW}=$ 127 ; transmission rate, $1 / \mathrm{T}=78.7 \mathrm{Kbits} / \mathrm{sec}$. A maximum of 71 chips delay spread has been considered and thus the intersimbolic interference will be negligible. The transmission scheme of figure (1), in which up to 6 pseudorandom sequences of maximum period theoretically mutually orthogonal, have been simulated, corresponding to five possible interfering users. Each user's sequence is multiplied by the symbol to be transmitted (BPSK modulated). Once the signals are modulated, they go through a selective frequency time variant channel. A different channel has been considered for each user. Value $L^{\prime}$ is computed as $L(L-1)$ where $L$ is taken as the mean of the paths generated at each impulse responses of the different users and it results a value of 15 paths. Considering that the variance of the arriving paths has been assumed to be the same for each channel $E\left[\alpha k^{2}\right]=0.12$, the results from figure (3) are obtained when only one interfering user is present. Figures (4) and (5) show the probability of error and its upper bound for three and five interfering users respectively and finally, figures (6) and (7) show only the upper bound for one, three and five interfering users for different signal to noise ratios.

\section{Conclusions}

Several interesting facts are obtained from the near-far effect in an optimum receiver under multipath operation conditions. These include the effect of the increase in the factor $\gamma$ and the number of interfering users on the BER, and, on the other hand, the limitation of the system capacity as function of the number users.

It is important to observe that small increases in the factor $\gamma$ can affect the BER in the event of multiple users access. In figure (8) for one interfering user, it can be observed than an increase of $\gamma$ can affect considerably the BER of the system. It should be taken into account that for the optimum detection, it is in a certain way desirable for the multipath problem, as the receiver, adapted to the channel of the mobile of interested, enhance the appropriate paths and discriminate the paths arriving from other PN sequences (diversity).

\section{Bibliography}

[1] M. Abramovitz, Handbook of Mathematical Functions, Dover publications, 1970

[2] B.R.Saltzberg, “ Intersymbol Interference Error Bounds with application to Ideal Bandlimited Signaling", IEEE Transactions on Information Theory, vol IT-14, $\mathrm{n}^{\circ} 4$, July 1968

[3] D.Borth and M.Pursley, " Analysis of Direct-Sequence Spread-Spectrum Multiple-Access Communications Over Rician Fading Channels ", IEEE Transactions on communications, vol. com-27, $n^{\mathrm{a}} 10$, October 1979

[4] E.Geraniotis, “ Direct-Sequence Spread-Spectrum MultipleAccess Communications Over Nonselective and FrequencySelective Rician Fading Channels", IEEE Transactions on communications, vol. com-34, $\mathrm{n}^{\mathrm{a}}$ 8, August 1986. 


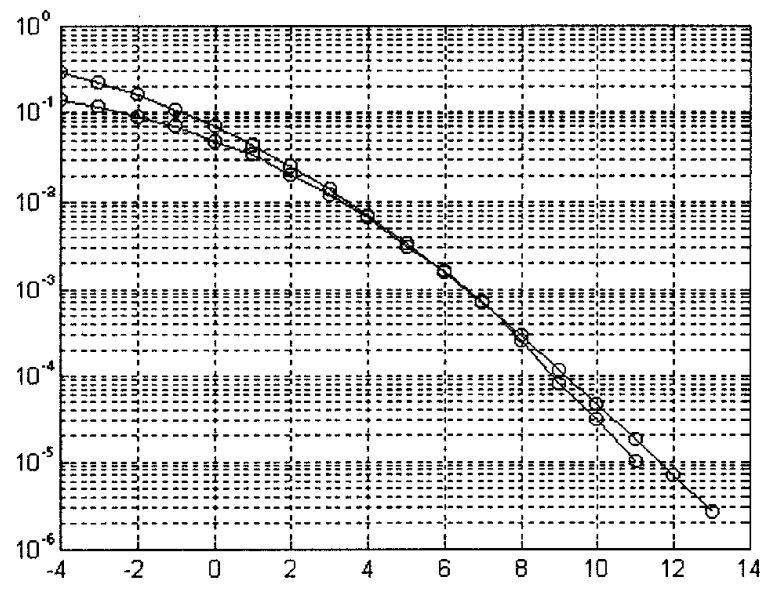

fig 3. Probability of error with one interfering user

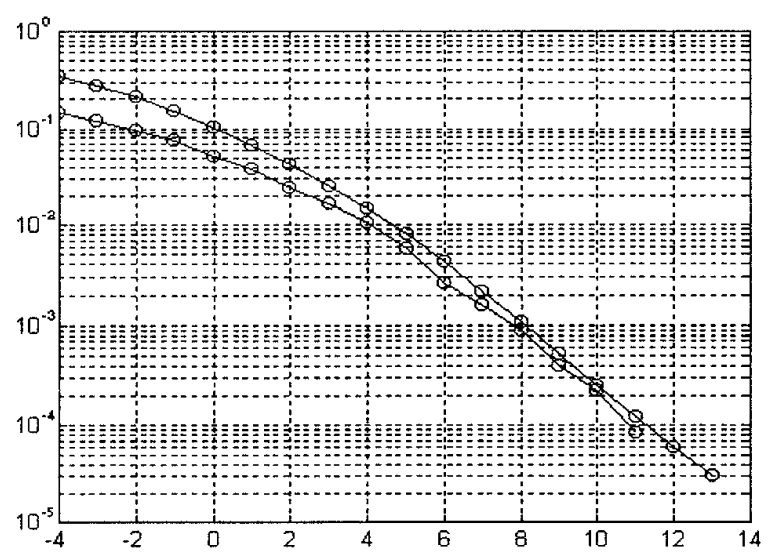

fig 5. Probability of error with five interfering users

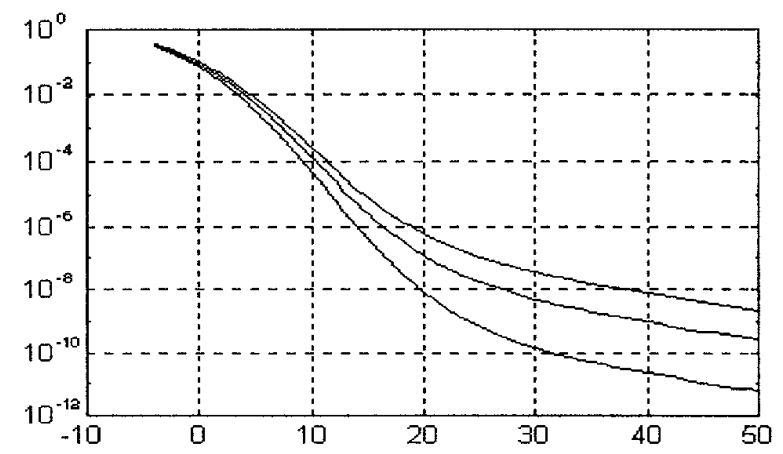

fig 7. Probability of error with 1,3 and 5 interfering users

[5] J.Lehnert and M.B.Pursley, "Multipath Diversity Reception of Spread-Spectrum Multiple-Access communications", IEEE Transactions on communications, vol. com-35, $n^{\mathrm{a}} 11$, November 1967

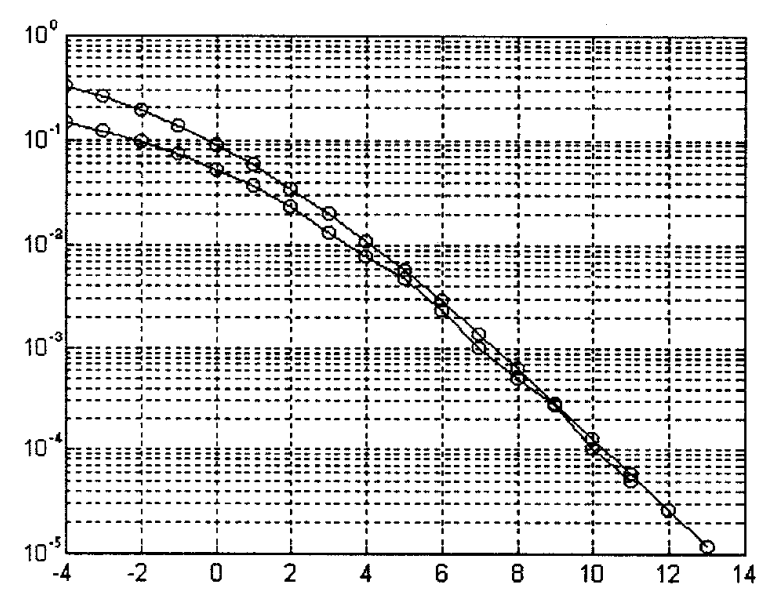

fig 4. Probability of error with three interfering users

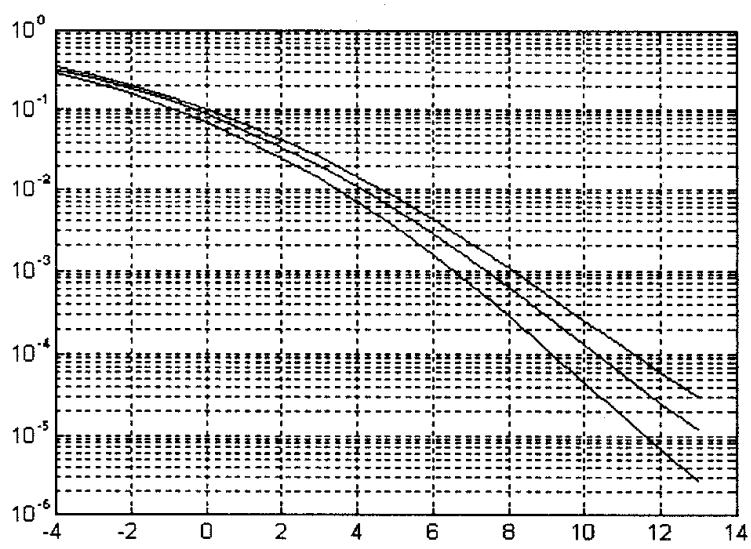

fig 6. Theoretic results

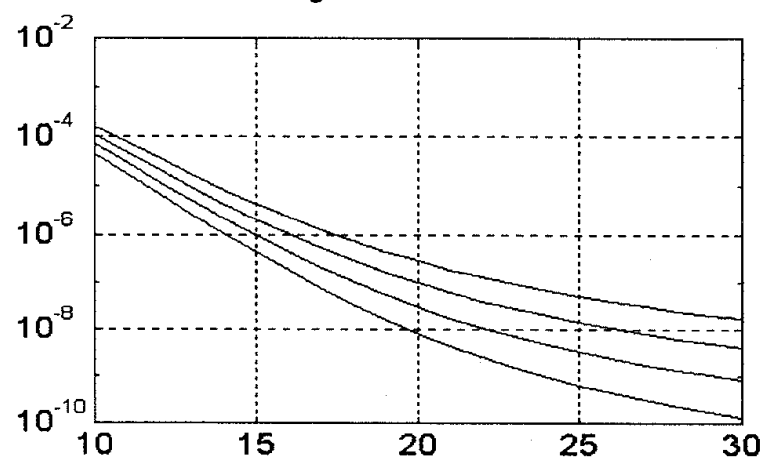

fig 8. Probability of error for one interfering user with $\gamma=0.14,0.16,0.18,0.20$ and $L^{\prime}=15$

[6] B.Glance and L.J.Greenstein, " Frequency-Selective Fading Effects in Digital Mobile Radio with Diversity Combining", IEEE Transactions on communications, vol. com-31, $\mathrm{n}^{\mathrm{a}} 9$, September 1983. 